\title{
BMJ Open Cohort Study of Pulmonary Tuberculosis (COSMOTB) identifying drug-resistant mutations: protocol for a prospective observational study in Korea
}

\author{
Jinsoo Min, ${ }^{1}$ Chaeuk Chung, ${ }^{2}$ Jinsook Lim, ${ }^{3}$ Jong Hyock Park, ${ }^{4}$ \\ Kyeong Seob Shin, ${ }^{5}$ Sung-Soo Jung, ${ }^{2}$ Ki Man Lee ${ }^{6}$
}

To cite: Min J, Chung C, Lim J, et al. Cohort Study of Pulmonary Tuberculosis (COSMOTB) identifying drugresistant mutations: protocol for a prospective observational study in Korea. BMJ Open 2018;8:e021235. doi:10.1136/ bmjopen-2017-021235

- Prepublication history for this paper is available online. To view these files, please visit the journal online (http://dx.doi. org/10.1136/bmjopen-2017021235).

Received 19 December 2017 Revised 3 August 2018 Accepted 22 August 2018
Check for updates

(C) Author(s) (or their employer(s)) 2018. Re-use permitted under CC BY-NC. No commercial re-use. See rights and permissions. Published by BMJ.

For numbered affiliations see end of article.

Correspondence to

Dr Ki Man Lee;

kimlee@chungbuk.ac.kr

\section{ABSTRACT}

Introduction Drug-resistant tuberculosis (TB) is a global concern. The proper diagnosis and management of drug-resistant TB are critical for improving treatment outcome. Molecular-based genotypic drug-susceptibility testing (DST) was developed to identify drug-resistant TB; however, discordant results from phenotypic and genotypic DST analyses have alarmed clinicians and raised concerns about the test's utility. Moreover, the characteristics of disputed mutations are not well studied and only based on retrospective study findings.

Methods and analysis We describe a 28-month prospective observational cohort study ongoing at two university-affiliated hospitals in South Korea. The cohort study will enrol and evaluate 600 adults with pulmonary TB. Relevant clinical and epidemiological data will be collected prospectively and participants will be evaluated at each hospital during anti-TB treatment to identify factors associated with TB treatment outcomes. Respiratory specimens will be collected at select visits. After generating a well-characterised cohort, patterns of drug resistance on both phenotypic and genotypic DSTs and associated mutations including the disputed mutation will be evaluated. We will also identify various clinical and socioeconomic factors that affect the causes of drug resistance and their clinical outcomes.

Ethics and dissemination The study protocol is approved by the Institutional Review Boards of Chungbuk National University Hospital and Chungnam National University Hospital. Study results will be disseminated through peerreviewed journals and conference presentations.

Trial registration number KCT0002594.

\section{INTRODUCTION}

Despite a growing economy and continuous national efforts to control tuberculosis (TB), South Korea has a disproportionately high burden of TB compared with other high-income countries. ${ }^{1}$ Despite a decrease in the overall incidence of $\mathrm{TB}$, surveys indicate that the incidence of drug-resistant TB is rising. Drug-resistant TB is a global concern and an

\section{Strengths and limitations of this study}

- This will be the first study to prospectively identify mutations associated with drug resistance in tuberculosis (TB).

- Discordant phenotypic and genotypic drug susceptibility test results and the associated disputed mutation will be identified, and their clinical outcome will be evaluated.

- This study will recruit and follow-up patients with pulmonary TB, from initial diagnosis to end of anti-TB treatment, using a standardised protocol in order to improve the quality of data collection.

- Only two hospitals are designated for this study, which limits generalisability of the study results to a larger population.

obstacle for the End TB Strategy proposed by the WHO. ${ }^{23}$

Conventional treatment of multidrug-resistant TB (MDR-TB) as recommend by the WHO is associated with a cure rate of approximately 50\%. In South Korea, several retrospective studies have indicated an MDR-TB treatment success rate of $44 \%-75 \% .^{5}$ The proper diagnosis and management of MDR-TB are critical for improving treatment success rates and patient outcomes.

Culture-based phenotypic drug-susceptibility testing (DST) has conventionally been used for the detection of drug resistance; however, the interval between sample collection and drug-susceptibility determination is a main drawback of culture-based DST. Recently, molecular-based genotypic DST, which detects genetic mutations in Mycobacterium tuberculosis that confer drug resistance, was developed as an adjunct to phenotypic DST. In the past decade, the WHO has endorsed the use of Xpert MTB/RIF for the 
diagnosis of active TB disease and line probe assays for rapid DST of anti-TB drugs. ${ }^{6} 7$ To this end, the implementation of genotypic diagnostic tools has considerably increased the efficiency and speed of drug-resistant TB detection. $^{8}$

Genes associated with isoniazid and rifampicin resistance ( $k a t G$ and $i n h A$ for isoniazid and $r p o B$ for rifampicin) are the genes most commonly screened by genotypic DST. ${ }^{9}$ Yet, not all mutations of these genes lead to drug resistance (eg, these genes can have silent mutations). Additionally, some disputed mutations are associated with discordant phenotypic and genotypic DST findings. Although several retrospective analyses have associated some of these disputed mutations with a poor prognosis compared with drug-sensitive $\mathrm{TB},{ }^{11}$ the characteristics and clinical manifestations of the disputed mutations are not well studied.

This cohort study of pulmonary TB is funded by the Korea Centres for Disease Control and Prevention in order to describe gene mutations associated with anti-TB drug resistance and identify their effects on treatment outcome. In this manuscript, we provide an overview of the design, methods and scope of this cohort study.

\section{METHODS AND ANALYSIS \\ Goals and objectives}

The overarching goal of this cohort study is to develop a well-characterised cohort of patients with pulmonary TB with robust clinical data and a comprehensive specimen repository. This cohort will address three primary objectives: (1) characterise patterns of drug resistance and identify associated gene mutations; (2) account for discordant results of phenotypic and genotypic DST; and (3) identify the clinical outcomes of disputed mutations.

\section{Study design and setting}

The study is a 28-month prospective observational cohort study that is ongoing at two university-affiliated hospitals in South Korea since October 2016. Chungbuk National University Hospital (CBNUH, Cheongju, South Korea) and Chungnam National University Hospital (CNNUH, Daejeon, South Korea) have private-public matrix (PPM) projects for $\mathrm{TB}$ control in South Korea ${ }^{12}$ and manage the PPM project in each respective region. $\mathrm{CBNUH}$ is a tertiary hospital in Chungbuk province that identifies 250 new cases of TB annually. CNNUH is a tertiary hospital in Daejeon metropolitan city that identifies 500 new cases of TB annually.

\section{Study population}

The TB cohort will include 600 adults with newly diagnosed pulmonary TB who visit CBNUH or CNNUH. The inclusion criteria are as follows: (1) age 19 years or older, (2) patients who start anti-TB treatment (ATT) following a diagnosis or suspicion of pulmonary TB and (3) patients with pulmonary TB who have been receiving ATT for less than 1 month.
Table 1 Study evaluations performed in the COSMOTB

\begin{tabular}{lll}
\hline $\begin{array}{l}\text { Socioeconomic } \\
\text { evaluation }\end{array}$ & Clinical evaluation & $\begin{array}{l}\text { Laboratory } \\
\text { evaluation }\end{array}$ \\
\hline $\begin{array}{l}\text { Socioeconomic } \\
\text { status }\end{array}$ & Symptoms & AFB smear \\
$\begin{array}{l}\text { Occupational } \\
\text { status }\end{array}$ & Medical history & AFB culture \\
$\begin{array}{l}\text { Educational } \\
\text { status }\end{array}$ & Vital signs & Culture-based DST \\
\hline $\begin{array}{l}\text { Smoking history } \\
\text { Alcohol history }\end{array}$ & Weight & Xpert MTB/RIF \\
& Middle upper & MTBDRplus \\
& arm circumference & DNA sequencing \\
& & $\begin{array}{l}\text { Minimal inhibitory } \\
\text { concentration }\end{array}$ \\
& & HIV testing \\
& & HBsAg/HBAb/Anti- \\
& & HCV \\
& & Complete blood \\
& & count \\
& & Blood chemistry \\
& &
\end{tabular}

AFB, acid-fast bacilli; DST, drug-susceptibility testing; HBV, hepatitis $B$ virus; $\mathrm{HCV}$, hepatitis $\mathrm{C}$ virus.

\section{Study procedures}

Relevant clinical and epidemiological data will be prospectively collected from enrolled patients using a case report form (tables 1 and 2). Participants will be evaluated at the hospital on study entry and at 2 weeks, 4 weeks, 2 months, 4 months, 6 months and 9 months after ATT initiation to identify factors associated with TB treatment outcomes. Respiratory specimens will be collected at select visits. All specimens will be decontaminated with $4 \% \mathrm{~N}$-acetyl-L-cysteine-sodium hydroxide and concentrated by centrifugation at $3000 \mathrm{~g}$ for $15 \mathrm{~min}$. Acid-fast bacilli (AFB) smear testing using light and fluorescent microscopy and Xpert MTB/RIF (Cepheid, Sunnyvale, California, USA) tests will be conducted at each hospital for rapid confirmation of $\mathrm{TB}$ and the detection of rifampicin resistance, respectively. Both Ziehl-Neelson and fluorescent staining using auramine-rhodamine are used for the AFB smear test at each hospital. Mycobacterium culture testing using both solid (3\% Ogawa media) and liquid (BACTEC MGIT 960 system, BD, New Jersey, USA) cultures will be performed at the reference laboratory (Green Cross Labcell, Yongin, South Korea).

Culture-based phenotypic DST and GenoType MTBDRplus (Hain Lifesciences, Nehren, Germany) will be performed on the samples positive for M. tuberculosis. Phenotypic DST will be determined by the absolute concentration method using Löwenstein-Jensen media as recommended by $\mathrm{WHO}^{13}$ at the supranational reference laboratory (Korean Institute of Tuberculosis, Osong, South Korea). The drugs and their critical concentrations for resistance are as follows: isoniazid $0.2 \mu \mathrm{g} / \mathrm{mL}$, rifampin 
Table 2 Study timeline of the COSMOTB

\begin{tabular}{|c|c|c|c|c|c|c|c|}
\hline & Enrolment & 2 weeks & 4 weeks & 2 months & 4 months & 6 months & 9 months \\
\hline Consent & \multicolumn{7}{|l|}{$x$} \\
\hline Medical history & $x$ & $x$ & $x$ & $x$ & $x$ & $x$ & $x$ \\
\hline Xpert MTB/RIF & \multicolumn{7}{|l|}{$x$} \\
\hline AFB smear & \multicolumn{2}{|l|}{$x$} & $\mathrm{X}$ & $x$ & $x$ & \multicolumn{2}{|l|}{$\mathrm{x}$} \\
\hline Drug-susceptibility testing & \multicolumn{7}{|c|}{$\begin{array}{l}\text { Culture-based phenotypic DST and MTBDRplus will be performed on samples positive for } \\
\text { mycobacterium. }\end{array}$} \\
\hline Chest image testing & $\mathrm{X}$ & $\mathrm{X}$ & $\mathrm{X}$ & $\mathrm{X}$ & $\mathrm{X}$ & $x$ & $\mathrm{X}$ \\
\hline HIV, HBV, HCV & \multicolumn{7}{|l|}{$x$} \\
\hline Complete blood count & $x$ & $x$ & $x$ & $x$ & \multicolumn{3}{|l|}{$x$} \\
\hline
\end{tabular}

AFB, acid-fast bacilli; DST, drug-susceptibility testing; HBV, hepatitis B virus; HCV, hepatitis C virus.

$40 \mu \mathrm{g} / \mathrm{mL}$, ethambutol $2.0 \mu \mathrm{g} / \mathrm{mL}$, streptomycin $10 \mu \mathrm{g} /$ $\mathrm{mL}$, amikacin $30 \mu \mathrm{g} / \mathrm{mL}$, kanamycin $30 \mu \mathrm{g} / \mathrm{mL}$, capromycin $40 \mu \mathrm{g} / \mathrm{mL}$, prothionamide $40 \mu \mathrm{g} / \mathrm{mL}$, cycloserine $30 \mu \mathrm{g} / \mathrm{mL}$, para-aminosalicylic acid $1.0 \mu \mathrm{g} / \mathrm{mL}$, ofloxacin $4.0 \mu \mathrm{g} / \mathrm{mL}$, levofloxacin $2.0 \mu \mathrm{g} / \mathrm{mL}$ and moxifloxacin $2.0 \mu \mathrm{g} / \mathrm{mL}$. Pyrazinamide susceptibility was determined by a pyrazinamidase test. ${ }^{14}$ The GenoType MTBDRplus, a reverse hybridisation line probe assay, will be performed in accordance with manufacturer specifications at the reference laboratory.

Subsequently, minimal inhibitory concentrations (MICs) for anti-TB drugs and DNA sequencing for genes for isoniazid and rifampicin resistance $(r p o B, k a t G$, inhA and $a h p C$ ) will be conducted at the National Institute of Health (Korea Centers for Disease Control and Prevention, Osong, South Korea). For determination of the MICs of anti-TB drugs, MYCOTB MIC plate (MYCOTB; Trek Diagnostic Systems, Thermo Fisher Scientific, Ohio, USA) will be used according to the manufacturer's instruction. Sanger sequencing will be adopted as a method of DNA sequencing of genes of drug resistance. PCR products will be sequenced using a 3500xl genetic analyser (Applied Biosystems, California, USA). All DNA sequences were assembled and compared with the M. tuberculosis $\mathrm{H} 37 \mathrm{Rv}$ reference sequence (GenBank NC_000962) by the CLC Main Workbench 7.6.4 (CLC bio Aarhus, Denmark).

\section{Specimen collection}

Sputum is the main respiratory specimen; however, bronchoscopy will be performed when a participant cannot expectorate adequate and suitable sputum for testing, and specimens from bronchoscopic aspirates will be substituted for sputum. Transtracheal aspirates will be used for participants who are intubated for mechanical ventilation. All collected specimens will be transferred to a reference laboratory as quickly as possible. When a transfer delay of more than 1 hour is anticipated, the specimen will be stored in a freezer at $4^{\circ} \mathrm{C}$. Respiratory specimens and $M$. tuberculosis isolates will be processed, aliquoted and stored in cryo containers.

\section{Anti-TB treatment}

All enrolled participants will initially receive a minimum of 6 months standard ATT, including combination isoniazid, rifampicin, ethambutol and pyrazinamide intensive therapy during the first 2 months followed by isoniazid and rifampicin continuation therapy for 4 months as recommended by the Korean National TB Guidelines. ${ }^{15}$

Given that genotypic DST can reveal drug-resistant mutations during standard treatment, the ATT regimen may be changed at the discretion of investigator for the following cases: (1) recurrent disease, (2) a history of treatment failure, (3) loss to follow-up, (4) immunocompromised status (eg, participants with HIV) and (5) patient with non-compliance. After changing the treatment regimen, the investigator will await the results of conventional phenotypic DST in order to confirm drug resistance.

We define treatment outcomes for drug-sensitive TB in accordance with the Korean National TB Guidelines as follows:

- Cured; a patient with pulmonary TB with bacteriologically confirmed $\mathrm{TB}$ at the beginning of treatment who was smear-negative or culture-negative in the last month of treatment and on at least one other previous occasion.

- Treatment completed; a patient with $\mathrm{TB}$ who completes treatment without evidence of failure but with no record of negative sputum smear or culture results in the last month and on at least one other previous occasion, either because tests were not completed or because results are unavailable. 
- Treatment failed: a patient with TB with a positive sputum smear or culture at month 5 or later during treatment.

- Died: a patient with TB who dies for any reason before or during the course of treatment.

- Lost to follow-up: a patient with TB who does not start treatment or whose treatment is interrupted for two consecutive months or more.

We divided treatment outcomes into two categories: (1) favourable outcome (the sum of patients classified as cured or treatment completed) and (2) unfavourable outcome (the sum of patients classified as treatment failed, died and loss to follow-up). For patients with rifampicin-resistant or MDR-TB, different definitions of cured, treatment completed and treatment failed will be adopted in accordance with the Korean National TB guidelines.

\section{Data analysis (statistical considerations)}

Patterns of drug resistance obtained from the DSTs will be evaluated and their associated mutations will be identified. After the confirmation of drug resistance, we will compare the results of phenotypic and genotypic DST. Gene mutations will be stratified into three categories: non-disputed mutations, disputed mutations and silent mutations. We will also identify and compare treatment outcomes as per the types of resistance gene mutations. Lastly, we will identify the clinical and socioeconomic factors that affect causes of drug resistance.

According to the annual TB report of South Korea, about 10000 patients with smear-positive pulmonary TB are notified each year. ${ }^{16}$ For the 28 -month study period, we estimate a study population of 23000 . The sample size was based on an anticipated $14 \%$ prevalence of drug-resistant TB among patients with smear-positive pulmonary TB in South Korea. ${ }^{17}$ Given an alpha level of 0.05 and a power of $95 \%$ CI, we calculated a required sample size of 184 using OpenEpi software V.3.01 (OpenEpi, Atlanta, Georgia, USA). The proportion of smear-positive pulmonary TB accounts for one-third of the total number of cases of pulmonary TB. Thus, we calculated a final sample size of 600 assuming an attrition rate of $10 \%$.

Data regarding patients, clinical characteristics and treatment outcomes will be reported as means, SD, frequency tables, rates and proportions. Comparisons of the clinical characteristics of pulmonary TB with drug resistance on both phenotypic and genotypic DSTs will be conducted using Pearson's $\chi^{2}$ or Fisher's exact tests for dichotomous variables and Student t-tests, paired t-tests and one-way analyses of variance for continuous variables. Multiple logistic regressions will be performed to identify risk factors associated with unfavourable outcomes. All significant variables in the univariate analysis including age and sex will be used in the multivariate analysis. A two-sided alpha level of 0.05 will be used as the threshold for statistical significance, and all analyses will be conducted using statistical software.

\section{Organisational oversight}

Before starting the cohort study, we will organise an administrative structure comprising a leadership group, a scientific working group, an operational working group and advisory boards. The leadership group will provide operational and scientific oversight. The scientific working group will contribute to intellectual productivity and output in their respective scientific disciplines. The operational working group will oversee the management of operations and logistics by performing periodic monitoring and evaluation processes throughout the course of the cohort study. The operational working group will hold monthly meetings at each study site to ensure performance by standardising the study protocol as well as the implementation of data collection instruments and operating procedures. The leadership group together with scientific and operation working groups will hold quarterly meetings to manage overall procedures for monitoring and quality control.

\section{Patient involvement}

Patients were not involved in setting the research question, the outcome measures, the design or implementation of the study. Patients will not be involved in the recruitment to and conduct of the study. We plan to translate the results into short, easy-to-read summaries and to disseminate it to the relevant patient community through local media.

\section{ETHICS AND DISSEMINATION}

The protocol and informed consent forms are approved for scientific content and compliance with human subject research regulations. All investigators and study personnel are trained in research ethics and human subjects' protection. The study principal investigator holds primary responsibility for the preparation of publications. The investigators will disseminate the findings of this research through publication in a peer-reviewed journal and via conference presentations. In compliance with the policy of the International Committee of Medical Journal Editors, this study was registered with the Clinical Research Information Service, Republic of Korea (cris. nih.go.kr, KCT0002594) in November of 2017.

\section{DISCUSSION}

As per the WHO's End TB Strategy, a 95\% reduction in the number of deaths from $\mathrm{TB}$ and a $90 \%$ reduction in the incidence of TB should be achieved by 2035 compared with 2015. ${ }^{3}$ An important component of this strategy is preventing the spread of drug-resistant TB. An accurate and reliable method for identifying drug resistance is paramount to the success of the END TB Strategy. A recent study ${ }^{18}$ investigated how different genotypic DST assays influence the design of anti-TB drug regimen among patients with MDR-TB, and revealed that the concordance of a genotypic DST assay with phenotypic 
DST can be deceptively high, and more than half of the drug in the resulting regimens would be prescribed inappropriately. Based on the results of the present cohort study, we expect to compare the results of phenotypic and genotypic DSTs and describe the clinical characteristics of drug-resistant mutations, providing clinical evidence for diagnostic guidelines for genotypic DST and treatment guidelines for TB with drug resistance mutations.

In addition, it is important to acknowledge that discrepancies of drug resistance observed between the phenotypic and genotypic methods are not exclusively due to problems related to the interpretation of genotypes. ${ }^{19}$ One of the attributed factors might be that phenotypic DST underestimates the true rate of drug resistance because some critical concentration is too high. Since MIC testing is one of our key methods for identifying drug resistance, we hope to be able to re-evaluate the critical concentrations to avoid systematic false-susceptible phenotypic DST results for a variety of first-line and second-line drugs.

The present study design has a few notable limitations. First, only two hospitals will be participating in this cohort study. Considering the hospital sizes and their roles in the area, it can be argued that our cohort will adequately represent the areas where the hospitals provide healthcare service; however, our results will not be generalisable to the larger population. We hope to successfully implement this cohort study and receive additional funds to expand the included regions. Second, we used the overall incidence of drug-resistant TB as a basis for our study calculation of sample size. Although the overall aim of our study is to identify mutations related to drug resistance in TB and their clinical implications, incidence of such mutations is not well known in South Korea. Thus, we had to calculate the sample size based on the incidence of phenotypically identified drug resistance, which may have caused some error in our sample size calculation. We hope to further explore this question at the end of our study. Third, there is no universal reference method for antimicrobial susceptibility testing in M. tuberculosis. ${ }^{19}$ In 2018, WHO published a technical report on critical concentrations for susceptibility testing of anti-TB drugs. ${ }^{20}$ Comparing proposed critical concentrations of fluoroquinolones and second-line injectables in this WHO report with those adopted in our study protocol, only moxifloxacin shows a different value of critical concentration. This WHO report proposes $1.0 \mu \mathrm{g} / \mathrm{mL}$ as the critical concentration of moxifloxacin, instead of $2.0 \mu \mathrm{g} / \mathrm{mL}$, which is used in our study protocol. Because our study protocol was designed in 2016, we could not adopt the new proposed value of critical concentration; however, MIC testing will be conducted for every isolate and compensate for this issue.

Despite these limitations, the cohort study described herein will be, to the best of our knowledge, the first study to prospectively identify mutations associated with drug resistance in TB. Standardised protocols, data collection and operating procedures will ensure the comparability and high quality of data across study sites.

\section{Author affiliations}

${ }^{1}$ Division of Pulmonary and Critical Care Medicine, Department of Internal Medicine, Daejeon St. Mary's Hospital, College of Medicine, The Catholic University of Korea, Daejeon, Republic of Korea

${ }^{2}$ Division of Pulmonary and Critical Care Medicine, Department of Internal Medicine, Chungnam National University Hospital, Daejeon, Republic of Korea

${ }^{3}$ Department of Laboratory Medicine, Chungnam National University Hospital, Daejeon, Republic of Korea

${ }^{4}$ Department of Health Informatics and Management, Chungbuk National University College of Medicine, Cheongju, Republic of Korea

${ }^{5}$ Department of Laboratory Medicine, Chungbuk National University College of Medicine, Cheongju, Republic of Korea

${ }^{6}$ Department of Internal Medicine, Chungbuk National University College of Medicine, Cheongju, Republic of Korea

Contributors JM drafted the first version of this manuscript. JM and KML conceived and designed the overall study. JM, CC, SSJ and KML are responsible for study oversight, management and coordination. JM, CC, JL, JHP, KSS, SSJ and KML reviewed the manuscript for intellectual content and approved the final version of the report.

Funding This work was supported by the Research Program funded by the Korea Centers for Disease Control and Prevention (2016E4600301).

Competing interests None declared.

Patient consent Obtained.

Ethics approval Institutional Review Boards of Chungbuk National University Hospital (2016-10-003) and Chungnam National University Hospital (2016-12-036).

Provenance and peer review Not commissioned; externally peer reviewed.

Open access This is an open access article distributed in accordance with the Creative Commons Attribution Non Commercial (CC BY-NC 4.0) license, which permits others to distribute, remix, adapt, build upon this work non-commercially, and license their derivative works on different terms, provided the original work is properly cited, appropriate credit is given, any changes made indicated, and the use is non-commercial. See: http://creativecommons.org/licenses/by-nc/4.0/.

\section{REFERENCES}

1. Kim JH, Yim JJ. Achievements in and challenges of tuberculosis control in South Korea. Emerg Infect Dis 2015;21:1913-20.

2. Jassal MS, Bishai WR. Epidemiology and challenges to the elimination of global tuberculosis. Clin Infect Dis 2010;50:S156-64.

3. Mariandyshev A, Eliseev P. Drug-resistant tuberculosis threatens WHO's End-TB strategy. Lancet Infect Dis 2017;17:674-5.

4. Dheda K, Gumbo T, Maartens G, et al. The epidemiology, pathogenesis, transmission, diagnosis, and management of multidrug-resistant, extensively drug-resistant, and incurable tuberculosis. Lancet Resp Med 2017;5:291-360.

5. Kang YA. Diagnosis and treatment of multidrug-resistant tuberculosis. J Korean Med Assoc 2014;57:27.

6. World Health Organization. Policy statement: automated real-time nucleic acid amplification technology for rapid and simultaneous detection of tuberculosis and rifampicin resistance: Xpert MTB/RIF system. Geneva, Switzerland: WHO, 2011.

7. World Health Organization. Molecular line probe assays for rapid screening of patients at risk of multidrug-resistant tuberculosis (MDRTB). Geneva, Switzerland: WHO, 2008.

8. Sachdeva KS, Raizada N, Sreenivas A, et al. Use of Xpert MTB/RIF in Decentralized public health settings and its effect on pulmonary TB and DR-TB case finding in India. PLoS One 2015;10:e0126065.

9. Domínguez J, Boettger EC, Cirillo D, et al. Clinical implications of molecular drug resistance testing for Mycobacterium tuberculosis: a TBNET/RESIST-TB consensus statement. Int J Tuberc Lung Dis 2016;20:24-42.

10. Rigouts L, Gumusboga M, de Rijk WB, et al. Rifampin resistance missed in automated liquid culture system for Mycobacterium tuberculosis isolates with specific rpoB mutations. J Clin Microbiol 2013;51:2641-5.

11. Van Deun A, Aung KJ, Hossain A, et al. Disputed rpoB mutations can frequently cause important rifampicin resistance among new tuberculosis patients. Int J Tuberc Lung Dis 2015;19:185-90.

12. Kim HJ, Bai G-H, Kang MK, et al. A Public-Private collaboration model for treatment intervention to improve outcomes in patients with tuberculosis in the private sector. Tuberc Respir Dis 2009;66:349. 
13. World Health Organization. Companion handbook to the WHO guidelines for the programmatic management of drug-resistant tuberculosis. Geneva, Switzerland: World Health Organization, 2014.

14. Wayne LG. Simple pyrazinamidase and urease tests for routine identification of mycobacteria. Am Rev Respir Dis 1974;109:147-51.

15. Korea Centers for Disease Control and Prevention. Korean guidelines for tuberculosis. 3rd edn. Osong, South Korea: KCDC, 2017.

16. Korea Centers for Disease Control and Prevention. Annual report on notified tuberculosis in Korea. Osong, South Korea: KCDC, 2016.

17. Bai GH, Park YK, Choi YW, et al. Trend of anti-tuberculosis drug resistance in Korea, 1994-2004. Int J Tuberc Lung Dis 2007;11:571-6.
18. Heyckendorf J, Andres S, Köser CU, et al. What Is Resistance? Impact of phenotypic versus molecular drug resistance testing on therapy for multi- and extensively drug-resistant tuberculosis. Antimicrob Agents Chemother 2018;62:e01550-17.

19. Schön T, Miotto P, Köser CU, et al. Mycobacterium tuberculosis drug-resistance testing: challenges, recent developments and perspectives. Clin Microbiol Infect 2017;23:154-60.

20. World Health Organization. Technical Report on critical concentrations for drug susceptibility testing of medicines used in the treatment of drug-resistant tuberculosis. Geneva, Switzerland: World Health Organization, 2018. 